\title{
Machines à deuil
}

Mourning Machines

\section{Jan Baetens}

\section{OpenEdition}

\section{Journals}

Édition électronique

URL : https://journals.openedition.org/recherchestravaux/3047

DOI : 10.4000/recherchestravaux.3047

ISSN : 1969-6434

Éditeur

UGA Éditions/Université Grenoble Alpes

\section{Édition imprimée}

ISBN : 978-2-37747-241-3

ISSN : 0151-1874

\section{Référence électronique}

Jan Baetens, « Machines à deuil », Recherches \& Travaux [En ligne], 97 | 2020, mis en ligne le 12 novembre 2020, consulté le 01 juillet 2021. URL : http://journals.openedition.org/recherchestravaux/ 3047 ; DOI : https://doi.org/10.4000/recherchestravaux.3047

Ce document a été généré automatiquement le 1 juillet 2021.

(c) Recherches \& Travaux 


\title{
Machines à deuil
}

\author{
Mourning Machines
}

Jan Baetens

« De plus, rien ne s'arrête à l'individuel. »

(Paul Nougé, La Conférence de Charleroi)

\section{La perte du deuil}

1 Le deuil est universel : ce serait prétentieux d'affirmer que seuls les humains - et non les animaux par exemple - sont capables de s'endeuiller. C'est aussi un phénomène historiquement, culturellement, anthropologiquement déterminé et variable - et sans doute plus fragile et vulnérable qu'on ne le pense, comme le montrent aujourd'hui l'érosion des rites traditionnels, forcément collectifs, et le glissement de plus en plus rapide vers des formes de deuil individuel, difficiles à partager ou à faire partager.

Du point de vue littéraire, cette mutation génère de nouvelles formes d'écriture, plus proches des sensibilités contemporaines. En même temps, il se jette aussi un certain discrédit sur les expressions conventionnelles - marquées par les codes sociaux du deuil et les genres qui l'informent -, soupçonnées d'un manque d'authenticité. Il en va ainsi des écrits classiques sur le deuil, où l'auteur ne prend pas nécessairement la plume pour donner voix à un sentiment personnel et subjectif mais pour se faire le porte-parole d'une expérience collective, voire d'une tradition purement littéraire, comme il en va de la poésie lyrique ou amoureuse précédant le romantisme, où le « je » du texte n'est pas automatiquement censé refléter le vécu intime et unique de l'auteur. La modernité - postmodernité comprise - ne veut plus d'une parole littéraire ou artistique qui ne soit pas l'émanation directe de l'expérience d'un individu et elle tend à rejeter les textes ou les styles basés sur l'imitation de règles et de genres codifiés.

Un tel rejet est idéologique, certes. Même à l'époque des autofictions triomphantes, nous n'admettons plus les textes qui «trichent» avec les sentiments. Mais il est également littéraire : nous n'arrivons plus à comprendre le fonctionnement de textes basés sur la dissociation radicale $d u$ «je » du texte, qui occupe tout le devant de la scène, et du « moi » de l'auteur, qui demeure une inconnue - et qui en bien des cas n'a 
aucune importance, pour ne pas dire aucune existence. Dans son étude de l'exclusion des textes et genres préromantiques de l'enseignement contemporain, Ingrid Riocreux démontre clairement à quel point les notions modernes d' « auteur » et d'« originalité » débouchent sur une extension transhistorique du lyrisme contemporain, comme expression de soi, qui rend littéralement illisibles, avant de les exclure des programmes, des écritures plus anciennes régies par un tout autre horizon d'attente ${ }^{1}$. Les textes anciens, ceux du lyrisme "froid» où l'auteur imite un code plus qu'il ne cherche à faire ressentir des sentiments privés, soit restent incompris (et risquent d'être délaissés), soit sont mal lus (et souffrent de mille et une erreurs d'une lecture anachronique, faite à la lumière des goûts du jour).

Dans les deux cas, l'appauvrissement est considérable, et les observations de Riocreux sur l'ode ou l'élégie s'appliquent également à la littérature du deuil. La tradition littéraire est ou bien laissée de côté, car jugée inauthentique et partant illisible, ou bien mal lue, en tant qu'expression d'un vécu individuel que nous croyons nécessaire de projeter sur le texte. En même temps, ce que Riocreux appelle le " choc des mentalités " est aussi une réalité culturelle et le simple retour en arrière n'est pas moins désirable que la censure, souvent involontaire, des codes désuets. Dans les pages qui suivent, j'aimerais discuter d'une forme d'écriture, mélangeant textes et images, qui esquisse - peut-être - un dépassement de ce conflit. L'œuvre en question, dont je tenterai de démontrer le caractère générique, réconcilie en effet les aspects collectifs du code et de la norme, d'un côté, et la dimension proprement individuelle du deuil, de l'autre.

\section{De l'infra-ordinaire au deuil}

Gérard Courant est l'un des cinéastes les plus prolifiques de l'histoire du cinéma - on a pu comparer sa production personnelle à celle de tout l'INA! Il est notamment bien connu pour ses courts portraits filmés, les « cinématons » (actuellement, cet ensemble comprend déjà plus de trois mille portraits). Une des séries auxquelles Gérard Courant continue à travailler depuis de nombreuses années est "Mes Villes d'habitation", inventaires filmés, classés par ordre alphabétique, des rues et des places des diverses villes où il a habité au cours de sa vie (Lyon, Valence, Saint-Marcellin, Dijon). Le troisième tome de cette collection, À travers l'univers, filmé à partir de 1983 et terminé en 2005 (mais la date de la sortie officielle est $2008^{2}$ ), est une œuvre qui s'inspire de l'esprit de l'infra-ordinaire et du désir de rendre compte de « ce qui revient chaque jour, le banal, le quotidien, l'évident, le commun, l'ordinaire, le bruit de fond, l'habituel ${ }^{3}$ ». Plus concrètement, l'auteur cite au début les quatre sources suivantes : Louis Calaferte, Satori ; On Kawara, Continuity/Discontinuity 1963/1979 ; Pierre-François-Léonard Fontaine, Journal 1799-1853. Tomes 1 et 2 ; Georges Perec, Espèces d'espaces.

6 À travers l'univers, qui en soixante-dix-neuf minutes montre 127 rues et 17 places de Saint-Marcellin, où Gérard Courant a habité de l'âge de un à neuf ans, est un film qui applique un petit nombre de contraintes toutes simples, mais scrupuleusement respectées. Le cinéaste montre d'abord un gros plan de la plaque de la rue ou de la place (un plan de quelques secondes, légèrement plus long si la plaque contient également quelques informations historiques), puis un plan fixe d'exactement vingt secondes filmé sans coupure ou reprise depuis l'une des extrémités de la rue ou de la place. De plus, Gérard Courant n'utilise que des sons directs ; il n'y a ni voix off, ni accompagnement musical. Le titre du film fait allusion aux ambitions réalistes et 
descriptives de l'œuvre, le portrait de la ville de Saint-Marcellin (Isère, actuellement environ 8000 habitants) étant suffisamment complet et varié pour que d'éventuels visiteurs venus de Mars puissent se faire une idée complète de ce qu'est la vie sur Terre (maisons, habitants, déplacements) ${ }^{4}$.

7 Le film qui en résulte est appelé "ciné-poème", et non pas "documentaire ", le montage par ordre alphabétique des rues d'abord, des places ensuite, provoquant des rencontres et parfois des chocs qui excèdent tout objectif sociologique ou descriptif. Quand bien même Gérard Courant ne s'explique pas sur le choix du terme, inattendu mais un peu vague, le fait de nommer son œuvre « ciné-poème » dispose à une certaine lecture, notamment à une lecture élégiaque fondée sur les ruptures entre l'époque où le cinéaste habitait Saint-Marcellin, celle où il est retourné à sa ville d'enfance pour y filmer les lieux, celle enfin de la projection de l'œuvre, fatalement coupée du temps et du lieu de l'enregistrement. Peu à peu, le regard du spectateur se fait du reste plus ouvert et plus attentif à la surcharge émotive de ces images qui, prises en elles-mêmes, ne recherchent aucun effet poétique ${ }^{5}$. Surprenant au début de l'œuvre, le caractère poétique du film éclate dans la coda, une sorte de post-générique de quelque trois minutes, construite autour d'une chanson de Barbara, "Mon enfance», qu'on entend intégralement pendant que défilent à l'écran, alternant selon un rythme «normal» qui n'a plus rien à voir avec la règle de composition du film même, des éléments de générique (comme des remerciements à « la Société des gens bien élevés ») et des photos, actuelles ou d'époque, de Saint-Marcellin, qui rendent explicites certains des événements tragiques liés à la Résistance ( a Au Docteur Carrier / 1899-1943 / assassiné par / la Gestapo / le 29 novembre »). Paratexte clausulaire du film, la chanson de Barbara reprend, recadre, redéfinit la marque générique donnée par le paratexte inaugural, le « ciné-poème » du début se révélant in fine un poème de deuil. "Mon enfance » est en effet moins une chanson sur le retour au pays natal qu'une lamentation sur les peines que provoque, au-delà de l'évocation des morts et de l'effacement du passé, un tel retour :

Il ne faut jamais revenir

aux temps cachés des souvenirs

du temps béni de son enfance.

Car parmi tous les souvenirs

ceux de l'enfance sont les pires,

ceux de l'enfance nous déchirent.

8 Tel qu'en lui-même, À travers l'univers n'est pas un film de deuil ou sur le deuil et on pourra objecter qu'il existe comme un écart entre l'œuvre et son paratexte, qui abuserait ici de son pouvoir d'orientation de la lecture. Toutefois, pareille observation ne rendrait pas service à l'œuvre même, qui fonctionne bel et bien comme une «machine à deuil », expression dont il convient maintenant de préciser un peu le sens.

\section{Rien à voir, tout à regretter}

9 Comment une succession de "vues » peut-elle se transformer chemin faisant en suite lyrique - car telle est bien la première métamorphose que subit À travers l'univers? Par quels moyens une œuvre qui renvoie si fortement aux premiers films des frères Lumière, dont la visée documentaire est souvent opposée à l'élan lyrique symbolisé par Méliès, donne-t-elle lieu à une réception où l'émotion finit par 
concurrencer le réalisme? Il faut examiner ici la combinaison très efficace d'un ensemble de réductions et d'une série d'ajouts.

Les 117 (pour les rues), puis 17 (pour les places) fragments du film de Gérard Courant, qui ne déroge jamais à la contrainte imposée, sont tous d'une simplicité, voire d'une pauvreté visuelle et thématique extrêmement voulue. Il y a d'abord la quasineutralisation de l'intérêt du sujet qui apparaît à l'écran. Dans À travers l'univers, la caméra ne montre jamais quoi que ce soit de remarquable. On voit une rue, parfois des passants, souvent du trafic, et strictement rien d'autre, à aucun moment, comme si la vie se suffisait à elle-même. De la même manière, la caméra reste invariablement fixe, sa position - à hauteur du regard d'un observateur hypothétique - est on ne peut plus banale, il n'y a pas d'effets de montage à l'intérieur des plans, Gérard Courant n'a pas cherché les belles vues (sauf peut-être tout à la fin, dans le dernier plan de l'œuvre). À cela s'ajoute que la qualité matérielle des images ne semble bénéficier d'aucune attention soutenue : les couleurs sont fades, la résolution est faible, le choc avec la bande-son, avec des sons directs souvent très bruyants, est constamment là. Enfin, on est frappé par le peu d'interaction entre personnages filmés et opérateur - une différence notable avec les vues des frères Lumière, où le public filmé, en tout cas en ce genre de circonstances anonymes et banales, ne perd presque jamais de vue la caméra. Il n'y a pas ici, à quelques exceptions près (celles des enfants, justement, moins familiers des usages du cinéma dans l'espace public ?), aucun « regard retourné », pour reprendre la belle expression de Livio Belloï ${ }^{\circ}$.

11 Tout se passe un peu comme si les plans filmés aspiraient à la neutralité - supposée, évidemment - d'une caméra de surveillance. Or, dès la première séquence, cette indigence rigoureusement maintenue est nuancée par un gros plan sur une plaque de rue (rue ou boulevard $\mathrm{x}$, impasse $\mathrm{y}$, chemin ou avenue $\mathrm{z}$, etc.), tantôt purement indicative (le nom de rue sert alors de "désignateur rigide ", comme diraient les linguistes : ce sont de purs noms propres, dont le sens s'épuise avec la référence à la chose désignée), tantôt sommairement explicative (quand il s'agit de lieux ou d'événements d'importance historique, la municipalité a fait apposer sous le nom de rue une brève explication didactique, pas toujours facile à comprendre pour les nonhistoriens du reste). La singularité des rues brise immédiatement le caractère interchangeable de la plupart des vues, souvent proches de l'anonymat, la vue de la rue $\mathrm{x}$ ne se distinguant qu'à grand-peine de celle de la rue y). Le changement essentiel vient toutefois du montage cinématographique, qui combine un critère temporel, presque de métronome (ladite contrainte des vingt secondes par plan de rue) et un principe sémantique, de possible solution de continuité d'une rue à l'autre (la contrainte du classement alphabétique n'assemble pas toujours ce qui se ressemble et peut coller bout à bout des éléments totalement disjoints, par exemple un pré et une rue commerçante). La ville de Saint-Marcellin n'est pas traversée comme le ferait un piéton, un cycliste ou un chauffeur, elle se trouve déclinée lettre par lettre, dans un tempo parfaitement immuable, avec des chocs qu'une approche plus réaliste serait incapable de faire surgir.

12 Ainsi retrouve-t-on les vertus du minimalisme: "Tout l'intérêt d'une activité minimaliste réside précisément dans son aptitude à focaliser l'attention sur les opérations effectuées et à dissuader les excursions fortuites ${ }^{7}$.» Or le minimalisme - enlèvement du superflu, concentration sur l'essentiel - n'est pas ici un but en soi, cela reste une technique qui pointe vers un contenu, vers une leçon: une certaine 
forme de quotidien, capable de basculer dans une forme de lyrisme (on ne rappellera jamais assez que Gérard Courant a préféré le terme de "ciné-poème » à toute référence à la tradition documentaire). L'approche minimaliste aidant, la description dégage une émotion de plus en plus forte. Techniquement, cette émotion est soutenue d'abord par le tempo envoûtant, ni trop lent, ni trop rapide, puis par la pure accumulation des vues, chacune d'elles presque indifférente mais bouleversante quand appréhendée en enfilade, mais aussi par les multiples surprises déclenchées à l'aide de l'arrangement alphabétique, aussi arbitraire que signifiant. Toutefois, la technique ne serait rien sans véritable contenu. S'agissant d'À travers l'univers, la densité sémantique de l'infraordinaire tient au contraste implicite, mais de plus en plus en visible, entre un jadis et un maintenant, entre ce qui a pu donner son nom à la rue et la rue telle qu'elle se présente maintenant (mais le hic et nunc de la caméra est un présent qui s'éloigne davantage du spectateur à chaque nouvelle projection, à chaque nouveau visionnement), entre la rue que le cinéaste a pu voir ou non dans son enfance et la rue vers laquelle il retourne adulte (et entre ce qu'il a pu vivre ou observer dans cette rue et ce qui a cessé d'y être présent tant d'années plus tard). L'insistance sur cet écart est discrète, mais non inexistante - et de ce point de vue, la fin de l'œuvre, qu'on évoquera plus loin, apporte sans doute une certaine surprise, mais ne représentera pas de véritable choc, tant la dimension mélancolique se loge dès les premiers plans au cœur des images. À cet égard, le label générique de «ciné-poème » s'avère absolument décisif : dès l'ouverture, le spectateur sait qu'il ne peut s'en tenir à la seule portée descriptive, purement visuelle d'À travers l'univers.

Plus le film montre les choses telles qu'elles sont, plus il fait ressentir l'absence de ce qu'elles ne sont plus. Or, cette impression très vive, celle de l'écart entre visible et invisible, présent et passé, dit et non-dit, est fonction de l'application stricte d'une série de règles qui, elles, sont à mille lieues de toute considération émotive ou psychologique (le métronome, l'alphabet, le plan fixe, l'évitement du spectaculaire, etc.). Et ces règles mêmes ne sont nullement séparables de la matérialité du médium qui les intègre. À travers l'univers est en effet une œuvre qui explore les enjeux les plus fondamentaux du cinéma, soit l'art des images fixes (les photogrammes) que le dispositif littéralement anime (la succession des images qui suscite l'illusion du mouvement). À l'instar de Chris Marker dans La Jetée $e^{8}$ mais dans une perspective radicalement opposée, Gérard Courant brouille les pistes entre animé et inanimé. Là où Marker montrait qu'il est possible de faire du cinéma à partir d'images photographiques, c'est-à-dire non mobiles 9 , Courant tend à réinscrire les images mobiles de son film dans le champ de la photographie, de manière à activer les facultés thanatographiques de l'image fixe, plus exactement de l'image photographique. Dans À travers l'univers, les plans de rue perdent un peu de leur caractère d'animation et se rapprochent de nouveau des premières vues Lumière, c'est-à-dire des photographies animées. Ce qu'on voit n'est pas une suite de photos qui se muent en séquence mobile, mais des séquences mobiles qui se figent mystérieusement en tableau vivant - quand bien même un tel tableau vivant, tel le modèle du peintre, peut toujours bouger.

Le cinéma fait donc du surplace, puis regarde en arrière, tel Orphée, pétrifiant ce qu'il aurait dû faire revivre sans le regarder. Le seul fait de regarder un film, où tout se passe soi-disant toujours au présent, dans un éternel présent, se transforme petit à petit en une expérience beaucoup plus douloureuse, traditionnellement jugée propre à la photographie, dont on dit qu'elle ne peut montrer la vie qu'après sa disparition. L'impression diffuse de deuil qui se dégage de l'œuvre de Gérard Courant précise ainsi 
sa base très complexe : le sens y intervient, indéniablement, mais aussi le travail sur les formes et une réflexion discrète mais poignante sur le médium.

\section{Quand le paratexte devient œuvre}

15 La fin du film réserve au spectateur une vraie surprise - mais, répétons-le : surprise n'est pas choc, la dimension poétique, élégiaque de l'œuvre étant programmée dès l'incipit. On y découvre la suite du générique initial, mais son traitement diffère absolument de ce qu'on avait vu au début du film, lui d'une sobriété exemplaire. La coda d'À travers l'univers ne peut donc pas être réduite au simple rang de paratexte. Elle fait d'autant plus partie intégrante du film, c'est-à-dire du «texte » cinématographique, qu'elle en bouleverse radicalement les données les plus fondamentales. Aguerri par le travail sur le montage, mais aussi par le respect scrupuleux des contraintes tout au long de l'œuvre proprement dite, le spectateur averti de Gérard Courant est comme forcé d'inclure cette « rallonge » au film même.

16 Mais comment les quelque trois minutes finales d'À travers l'univers changent-elles le film? En termes de contenu, il ne semble pas y avoir de rupture: les éléments y complètent ceux du générique initial, la chanson de Barbara et les photos de SaintMarcellin se présentent comme une variation sur le sujet de l'œuvre, soit le retour à la ville où l'auteur a passé huit années de son enfance. Mais mis à part ces données thématiques, tout change. Les images mobiles sont remplacées par des images fixes, des photos anciennes ou modernes de la ville de Saint-Marcellin. De plus, ces images ont subi un traitement chromatique très voyant. Le tempo de leur défilement s'accélère considérablement, la durée de leur présence à l'écran n'étant plus régie par la contrainte temporelle des vingt secondes par plan. Enfin, les photos ne sont plus "ancrées", comme aurait dit Roland Barthes, par une quelconque légende, en l'occurrence celle du nom de la rue ou de la place. Elles sont convoquées l'une après l'autre pour illustrer une idée générale de Saint-Marcellin, à la fois " ville d'habitation » et ville marquée par les événements tragiques liés à l'occupation nazie. Quant à la chanson de Barbara, dont la durée et le rythme déterminent la longueur et l'organisation interne de la coda, elle rompt de plusieurs manières avec les protocoles étroitement surveillés de l'œuvre. Tout à coup la bande sonore renonce au son direct et passe à un accompagnement musical non diégétique (la chanson est certes liée au thème fondamental du film, mais la chanteuse en est tout à fait absente). Enfin, la qualité acoustique de la chanson est beaucoup plus soignée que l'enregistrement brut des bruits de rue.

17 Le contraste est donc total et il constitue la preuve par neuf de ce que le film même avait préparé : sous les pavés, non pas la plage, mais la mort. La mélancolie croissante qu'avait engendrée la déclinaison des séquences de rue, puis de place, et la sensation de plus en plus prégnante que le retour au passé, même limité à l'observation étriquée de son état présent, est source de tristesse, pour ne pas dire de désespoir, tous ces éléments sont articulés de manière on ne peut plus explicite dans la coda de l'œuvre, que le spectateur reçoit comme un coup de poignard non pas dans le dos, car tout spectateur attentif s'attend confusément à une telle apothéose, mais dans le cœur.

18 Toutefois, le passage du deuil implicite du film au deuil explicite de la coda n'est pas le passage d'un sentiment de malaise encore mal défini à une forme de deuil intime ou personnel, Gérard Courant finissant par jouer cartes sur table. C'est plutôt la traduction 
de ce sentiment en quelque deuil presque impersonnel, en quelque vérité générale dissociée des référents précis en jeu. Le film cesse d'être un film sur Gérard Courant et Saint-Marcellin pour devenir un film sur tout le monde et son enfance perdue. L'on comprend mieux dès lors ce qui donne sa force émotive à la première partie d'À travers l'univers, à savoir les 117 et 17 plans de rues et de places, d'une austérité tranquille. Le film ne cherche pas à concurrencer la radicalité des avant-gardes des années $60^{10}$ et moins encore à flatter le goût snob des productions acclamées parce qu'ennuyeuses ${ }^{11}$ - il faut le redire : À travers l'univers est un film accessible et tout sauf ennuyeux. Le travail de Gérard Courant affirme sereinement l'immense pouvoir émotif de l'écriture à contrainte, pour peu qu'elle s'applique bien sûr à un sujet qui s'y prête (la litanie des "Je me souviens" de Georges Perec en serait un exemple très proche), et la capacité d'un tel travail à concilier l'objectivité et l'impersonnalité d'un code ou d'un protocole à l'investissement psychologique des individus autant que des communautés. C'est que la contrainte, avant d'être une technique capable de soutenir les auteurs en panne d'inspiration - idée diversement soulignée par Raymond Roussel dès 1935, puis par Raymond Queneau et bien d'autres oulipiens (dont le cofondateur du groupe, François Le Lionnais ${ }^{12}$ ) - est une démarche susceptible de créer une communauté. On se rappelle le fameux incipit de Comment j'ai écrit certains de mes livres, qui assimile communication et don :

Je me suis toujours proposé d'expliquer de quelle façon j'avais écrit certains de mes livres (Impressions d'Afrique, Locus Solus, l'Étoile au front et la Poussière de Soleils).

Il s'agit d'un procédé très spécial. Et, ce procédé, il me semble qu'il est de mon devoir de le révéler, car j'ai l'impression que des écrivains de l'avenir pourraient peut-être l'exploiter avec fruit ${ }^{13}$.

De ce point de vue, une «bonne» contrainte n'est pas seulement une contrainte révélée ou rendue lisible, c'est également une contrainte suffisamment souple pour se prêter à une facile appropriation. Une contrainte dissimulée ou une contrainte trop dure empêche en effet la création d'une communauté d'auteurs-lecteurs ou de lecteursauteurs, qui représente l'un des traits les plus spécifiques de la poétique de la contrainte, qui allie technique et sociabilité des processus d'écriture ${ }^{14}$. Cette possibilité d'une communauté, qui est au cœur de toute démarche fondée sur la contrainte, rejoint l'une des dimensions les plus fondamentales du langage humain, à savoir le caractère ouvert, contextuellement défini et redéfini, par un certain nombre de signes que Benveniste appelle shifters ${ }^{15}$. Des mots comme je, ici, maintenant changent de référent à chaque nouvel usage et les usagers peuvent se les échanger. Les pronoms du «discours»-les première et deuxième personnes, que Benveniste oppose à la troisième personne du "récit » - n'appartiennent pas à ceux qui les emploient, mais peuvent être investis à tour de rôle par les partenaires d'un dialogue, le je d'un premier énoncé devenant le tu d'un second, et vice versa.

\section{De l'empathie à l'appropriation}

Dans À travers l'univers la présence du deuil est forte. Certes, ce n'est ni un film sur le deuil, ni un film de deuil en soi. C'est un film qui construit la possibilité d'un deuil, pour lui-même comme pour le spectateur. Mais comment l'œuvre jette-t-elle les bases d'une telle interprétation? La description de Saint-Marcellin, pour objective et transparente qu'elle paraisse, crée une tension entre présent et passé, qui fait affleurer petit à petit la perte de ce dernier. Le thème du deuil a beau être absent de la surface de l'œuvre, il 
n'en informe pas moins sa structure en profondeur. Et plus on avance dans le film, plus cette absence du passé se fait lancinante. Dans un deuxième temps, tout à la fin, le thème du deuil se trouve comme déplacé du fait même qu'il est nommé. Le retour au lieu de l'enfance envahit soudainement le film dans sa coda, avec la chanson de Barbara et les images anciennes, mais ce retour est doublement détaché de l'enfance de Gérard Courant à Saint-Marcellin.

21 Le je qui parle n'est pas le je du réalisateur, le village d'antan vaut pour n'importe quel village d'antan. Dit autrement: au moment où le film passe du portrait de ville, à dominante sociologique ou documentaire, à la confession autobiographique, tant la ville que l'auteur accèdent au rang de termes génériques: Saint-Marcellin devient n'importe quelle ville; Gérard Courant, par Barbara interposée, devient n'importe quelle personne qui se souvient. Ce qui aurait pu être l'expression d'un deuil personnel prend ainsi un caractère universel. Le spectateur peut se reconnaître dans la chanson de Barbara, dont le rapport personnel très fort à la ville qu'elle chante n'a au fond plus aucune importance. Pour faire jouer la charge émotive de la chanson, il n'est plus nécessaire de savoir que la famille d'origine juive de l'artiste avait dû se réfugier à Saint-Marcellin dans les dernières années de la guerre. La force des shifters autorise le transfert du deuil de l'artiste à son public. De la même façon, et on revient ici au film proprement dit, il n'est pas nécessaire que le spectateur sache qui est Gérard Courant ou qu'il connaisse la ville de Saint-Marcellin. Telle est la force de nombre d'écritures à contrainte, que l'œuvre de départ cesse rapidement d'appartenir à son auteur original. Beaucoup d'auteurs à contrainte stimulent le réemploi de leurs techniques par autrui et il n'en va pas autrement dans le cas du film sur Saint-Marcellin. Il suffit de comprendre comment Gérard Courant a filmé, puis monté Àtravers l'univers, pour que chaque spectateur puisse inventer sa propre variation sur l'œuvre.

L'essentiel est bien là, dans ce croisement d'une des propriétés les plus fondamentales du langage humain et de la confiance donnée à une technique de composition. C'est la combinaison de l'écriture à contrainte et de l'utilisation de shifters qui explique la transformation d'un deuil individuel, celui qu'évoque le film de Gérard Courant, en deuil collectif, plus exactement en deuil que n'importe quel spectateur peut considérer comme sien. Car ce n'est pas uniquement la contrainte que le spectateur peut s'approprier - il lui est très facile de refaire pour sa propre ville ce que le cinéaste d'À travers l'univers a fait pour Saint-Marcellin -, c'est aussi le texte de Barbara qui reste ouvert à la reprise par le public. En s'interdisant d'identifier les shifters de la chanson, puisque le je qui chante ne se nomme pas Barbara, l'ici du retour n'est pas Saint-Marcellin et le maintenant du texte n'est pas 1968 (date de sortie de l'album contenant « Mon enfance »), l'artiste permet à l'auditeur d'occuper la place « vide » du je-ici-maintenant.

Dans À travers l'univers, ces deux mécanismes se complètent et se renforcent mutuellement, et c'est cela qui fait de cette œuvre une « machine à deuil », c'est-à-dire une œuvre dont le deuil d'une seule personne peut devenir le deuil personnel de tout spectateur. Ce mouvement ne consiste pas à transformer un deuil individuel en deuil collectif, c'est-à-dire de groupe ou de communauté, mais bel et bien à multiplier le deuil personnel, chaque membre individuel du public ayant la possibilité de faire surgir une forme de deuil qui lui est propre sans pour autant minimiser ou instrumentaliser le deuil contenu dans l'œuvre de départ. Une machine à deuil est une machine 
littéralement contagieuse, non une machine qui prend appui sur un geste de sympathie ou d'empathie, qui suppose inévitablement une distance, si réduite soit-elle.

Vue sous cet angle, la machine à deuil, qui tresse un pôle purement objectif, avec sa mise en sourdine de la parole autobiographique et son traitement longtemps implicite du thème du deuil, et un pôle fortement subjectif, avec son invitation au spectateur à endosser le deuil de quelque chose qui lui est à l'origine totalement étranger, pourrait constituer un dépassement du «choc entre univers mentaux", pour reprendre l'expression d'Ingrid Riocreux. On y trouve en effet, intimement mêlés, un travail sur des éléments parfaitement conventionnels, socialement codés, comme la contrainte et le discours, d'une part, et la possibilité de faire surgir un deuil à la fois universel et inaliénablement individuel, d'autre part. De telles machines ne restent pas sans effet sur nos approches du deuil. D'une part, il faut espérer qu'elles aideront à revenir sur des expressions plus anciennes, largement soumises à l'utilisation de codes qui ne demandent pas que l'auteur s'identifie à la voix du texte. Pensons par exemple à toutes les oraisons funèbres écrites sur commande. D'autre part, et s'agissant des productions contemporaines, elles devraient nous inciter à ne pas condamner comme faux ou inauthentique le travail sur la forme. Valéry, qui a généralement raison dans son combat contre les hypocrisies littéraires, a peut-être tort quand il reproche à Pascal de trop penser à l'effet rhétorique de sa terrible phrase sur le silence éternel des espaces infinis ${ }^{16}$.

\section{NOTES}

1. I. Riocreux, "L'Illisibilité en pratique ou le choc des univers mentaux (l'exemple du lyrisme) », dans J. Baetens et É. Trudel (dir.), «Crises de lisibilité », Fabula-LhT, n 16, 2016 [en ligne] <http:// www.fabula.org/lht/16/riocreux.html> (consulté le 27 juin 2017).

2. G. Courant, À travers l'univers (79 min.), s. l., production Gérard Courant, 2008. La version «complète» ou «intégrale» $d u$ film est en ligne sur Dailymotion: <https:// www.dailymotion.com/video/x221mhe_a-travers-l-univers-2005-de-gerard-courant_shortfilms> (consulté le 27 juin 2017). À noter que la version disponible sur YouTube du même film se trouve amputée d'une partie essentielle (la « coda », dont il sera question plus loin dans cette analyse).

3. G. Perec, L'Infra-ordinaire, Paris, Seuil, coll. «La librairie du xxI siècle », 1989 (texte de présentation du volume en quatrième de couverture).

4. Cela est répété par le cinéaste même dans son introduction au film projeté à la Cinémathèque de Lisbonne le 23 juin 2017, où j'ai eu la chance de découvrir et le cinéaste et son œuvre.

5. Pour une réflexion générale sur le poétique «hors poésie», voir «Un je-ne-sais-quoi de "poétique" ", N. Cohen et A. Reverseau (dir.), Fabula-LhT, nº 18, 2017 [en ligne] <http:// www.fabula.org/lht/18/> (consulté le 27 juin 2017).

6. L. Belloï, Le Regard retourné. Aspects du cinéma des premiers temps, Montréal/Paris, Éditions Nota Bene/Klincksieck, 2002.

7. J. Calvelo et P. Hamel, « Géographismes », Conséquences, n 9, 1986, p. 46-47. 
8. C. Marker, La Jetée, Paris, Argos Films, 1962. Il existe de ce film, labellisé «photo-roman » par son auteur, une version (bilingue) sous forme de "ciné-roman-photo » réalisé en 1992 par le typographe Bruce Mau pour le compte des éditions Zone Books à New York.

9. On sait qu'à une exception près, tous les plans de La Jetée sont faits d'images fixes.

10. R. Krauss, " "A Voyage on the North Sea": Art in the Age of the Post-Medium Condition ", Londres, Thames and Hudson, 1999.

11. Pour une analyse « cadrée » de la valorisation de l'ennui dans une esthétique plus générale du spectateur du cinéma, voir L. Jullier, Qu'est-ce qu'un bon film ?, Paris, La Dispute, 2009.

12. F. Le Lionnais, «La Lipo (Le premier manifeste) », dans Oulipo, La littérature potentielle, Paris, Gallimard, coll. « Idées », 1973, p. 19-22.

13. R. Roussel, Comment j'ai écrit certains de mes livres [1935], Paris, U.G.E., coll. «10-18», 1977, p. 11.

14. J. Tabbi, «Electronic Literature as World Literature; or, The Universality of Writing under Constraint », Poetics Today, vol. 31, n 1, 2010, p. 17-50.

15. É. Benveniste, Problèmes de linguistique générale, Paris, Gallimard, 1966 (voir les chapitres de la cinquième partie, consacrés à l'homme dans le langage).

16. P. Valéry, Euvres, I, Paris, Gallimard, coll. « Bibliothèque de la Pléiade », 1957, p. 458.

\section{RÉSUMÉS}

Le présent essai analyse le traitement du deuil à travers l'exemple d'un film de Gérard Courant, À travers l'univers (2008). Le réalisateur y filme le retour à un lieu doublement marqué par son vécu personnel et l'histoire avec une grande hache, Saint-Marcellin, dans un esprit qui s'inspire de l'esthétique de l'infra-ordinaire et de l'écriture à contrainte. Apparemment, le film se contente de montrer en soixante-dix-neuf minutes les 127 rues et 17 places de la ville (d'abord la plaque qui les identifie, puis un long plan fixe d'exactement vingt secondes pris depuis l'une des extrémités de la rue ou de la place). Peu à peu, un malaise s'installe, que la chanson finale de Barbara, Mon enfance, permet de situer dans sa juste perspective, celle du deuil.

This essay analyzes the treatment of mourning through the analysis of a film by Gérard Courant, À travers l'univers (2008). In this movie, the director displays his return to a place that has a strong autobiographical as well as historical tone for him and his country, Saint-Marcellin, and he does so in a style that is inspired by the aesthetics of the infra-ordinary as well as constrained writing. At first sight, the seventy-nine minutes of the movie seem to show nothing else than the 127 streets and 17 places of the city (Courant first shows the street sign before continuing with a 20 seconds still shot of the street or the place seen from one of its ends). An uncanny feeling progressively comes to the fore and the song by Barbara, Mon enfance, helps to reframe the whole picture in its fundamental tone of mourning. 
INDEX

Mots-clés : Gérard Courant, Saint-Marcellin, Barbara, infra-ordinaire, écriture à contrainte, cinéma

Keywords : Gérard Courant, Saint-Marcellin, Barbara; infra-ordinary, constrained writing, cinema

\section{AUTEUR}

\section{JAN BAETENS}

Jan Baetens est professeur d'études culturelles à l'université de Leuven, où il travaille essentiellement sur la poésie contemporaine et les rapports entre textes et images dans les genres dits mineurs (bande dessinée, novellisation, roman-photo). Il a récemment publié Adaptation et bande dessinée (Les Impressions nouvelles, 2020) et Une fille comme toi (JBE éditions, 2020), un remix de sa collection personnelle de ciné-romans-photos. 